\title{
Manufacture and Cytotoxicity of a Lead-free Piezoelectric Ceramic as a Bone Substitute-Consolidation of Porous Lithium Sodium Potassium Niobate by Cold Isostatic Pressing
}

\author{
Qi Wang ${ }^{1,3}$, Jun Yang ${ }^{2}$, Wu Zhang ${ }^{3}$, Roxanne Khoie ${ }^{3}$, Yi-ming Li $^{3}$, Jian-guo Zhu ${ }^{2}$, Zhi-qing Chen ${ }^{1 *}$ \\ ${ }^{1}$ State Key Laboratory of Oral Diseases, West China College of Stomatology, Sichuan University, Chengdu, China \\ ${ }^{2}$ College of Materials Science and Engineering, Sichuan University, Chengdu, China \\ ${ }^{3}$ Center for Dental Research, School of Dentistry, Loma Linda University, Loma Linda, USA
}

\begin{abstract}
Qi Wang, Jun Yang, Wu Zhang, Roxanne Khoie, Yi-ming Li, Jian-guo Zhu, Zhi-qing Chen. Manufacture and Cytotoxicity of a Lead-free Piezoelectric Ceramic as a Bone Substitute - Consolidation of Porous Lithium Sodium Potassium Niobate by Cold Isostatic Pressing. International Journal of Oral Science, 1(2): 99-104, 2009
\end{abstract}

Aim The piezoelectric properties and cytotoxicity of a porous lead-free piezoelectric ceramic for use as a direct bone substitute were investigated.

Methodology Cold isostatic pressing (CIP) was applied to fabricate porous lithium sodium potassium niobate $\left(\mathrm{Li}_{0.06} \mathrm{Na}_{0.5} \mathrm{~K}_{0.44}\right) \mathrm{NbO}_{3}$ specimens using a pore-forming method. The morphologies of the CIP-processed specimens were characterized and compared to those of specimens made by from conventional pressing procedures. The effects of the ceramic on the attachment and proliferation of osteoblasts isolated from the cranium of 1-day-old SpragueDawley rats were examined by a scanning electron microscopy (SEM) and methylthiazol tetrazolium (MTT) assay.

Results The results showed that CIP enhanced piezoelectricity and biological performance of the niobate specimen, and also promoted an extracellular matrix-like topography of it. In vitro studies showed that the CIP-enhanced material had positive effects on the attachment and proliferation of osteoblasts.

Conclusion Niobate ceramic generated by CIP shows a promise for being a piezoelectric composite bone substitute.

Keywords lead-free piezoelectric ceramic, cold isostatic pressing (CIP), cytocompatibility, osteoblast

Document code: A $\quad$ CLC number: TB39 Received Mar. 2, 2009; Revision accepted Mar. 30, 2009

\section{Introduction}

A variety of biomaterials for repairing bone defects have significantly increased since lead-free piezoelectric ceramics were introduced (Feng et al., 1997) on account of their stable piezoelectricity. There is evidence that stress-generated potentials produced by piezoelectric ceramics can stimulate bone regeneration in situ. For example, barium titanate implants promoted osteogenesis in dog femurs (Park et al., 1981). Lithium sodium potassium niobate, a new generation of lead-free piezoelectric ceramics, has expanded its scope of reperaing bone defects owing to its better and more stable piezoelectricity compared with barium titanate (Saito et al., 2004). Studies have shown that alkaline niobate is able to enhance the deposition of hydroxyapatite and formation of a mineralized matrix (Wang, 2006). Furthermore, based on the homogenization theory, a "smart system" consisting of an inert perforated piezoelectric matrix filled with living osteoblasts may help the process of bone regeneration (Miara et al., 2005).

The idea of a porous lead-free piezoelectric ceramic was founded on these theories. Previously, we reported a fabrication method for making a biologically stable lead-free piezoelectric ceramic with pores and wetability favorable for nutrient exchange and osseointegration (Wang et al., 2008). Two particulate pore-forming agents (PFAs), ammonium oxalate monohydrate (AOM) and poly (methyl methacrylate) (PMMA), were compared for the 
fabrication of the porous lithium sodium potassium niobate (LNKN). We found that AOM was a better porogen than PMMA in producing ceramics with a range of porosity between $30 \%$ and $50 \%$ (Wang et al., 2008).

To expand on previous findings, in this study we aimed to evaluate the pressure changes during sample preparation by cold isostatic pressing (CIP) and the effects of CIP on piezoelectricity and biological performance. The piezoelectric strain factor $\left(d_{33}\right)$ and the electromechanical coupling factor $\left(K_{\mathrm{p}}\right)$ were measured as parameters of piezoelectric properties. Furthermore, the attachment and proliferation of osteoblasts were examined as measures of biological behavior by using scanning electron microscopy (SEM) and the methylthiazol tetrazolium (MTT) assay.

\section{Materials and methods}

\section{Ceramic preparation}

The properties of the starting materials are given in Table 1. LNKN powder was mixed with $5 \%$ (mass \%) aqueous poly vinyl alcohol (PVA) as a binder. AOM was added to the LNKN powder in a proportion of $50 \%$ or $60 \%$ (volume \%), as fullydensed LNKN. The mixture was then ball-milled without alcohol. Large particles of the AOM powders were screened out using standard stainless steel sieves. The powder mixtures were divided into two groups with one group diepressed at $20 \mathrm{MPa}$ (Chen et al., 2007) and another at $100 \mathrm{MPa}$ (CIP, LDJ200/ 600-300I, Shanxi Machine Works, China) in a mold of $15 \mathrm{~mm}$ in diameter and $1 \mathrm{~mm}$ in thickness. Specimens of each composition were then sintered in air at $1075^{\circ} \mathrm{C}$ for 2 hours in a loosely covered $\mathrm{Al}_{2} \mathrm{O}_{3}$ crucible to ensure complete burnout of the porogens. Bulk density $\left(\rho_{\mathrm{B}}\right)$ was determined by Archimedes' method in which: porosity $(P)$ was calculated from the ratio of the bulk density to the theoretical density $\left(\rho_{\mathrm{Th}}\right)$ using the following equation:

$$
P=\frac{\rho_{\mathrm{Th}}-\rho_{\mathrm{B}}}{\rho_{\mathrm{Th}}} \times 100 \%
$$

The constitution and microstructure of the samples were examined by X-ray diffraction (XRD) (X'Pert Pro MPD, Philips, Holland) and SEM (S-2460N, Hitachi, Japan), respectively.

\section{Piezoelectric properties}

The specimens were polarized under a dc electric field of $2.2-2.4 \mathrm{kV} / \mathrm{mm}$ in air for 50 minutes at $20-25^{\circ} \mathrm{C}$ using copper sheets as electrodes tightly clamped to the flat surfaces. $d_{33}$ was measured with a model $d_{33}$ piezometer (ZJ-3A, China Science and Technology Institution, China). $K_{\mathrm{p}}$ was measured using an impedance analyzer (PV70A, Jiangsu Instrument Company, China). All three specimens were tested for each composite, and an average of at least six measurements over the surface of each was taken.

\section{Biological analyses}

\section{Cell culture}

Osteoblasts isolated from the cranium of 1-dayold Sprague-Dawley rats (SDO, West China Animal Center, China) were cultured routinely at $37^{\circ} \mathrm{C}$ in a humidified atmosphere of $5 \% \mathrm{CO}_{2}$, and maintained in F12 (Gibco Life Technologies, USA) supplemented with $10 \%$ fetal bovine serum (FBS; Hyclone Co., USA), $50 \mathrm{IU} / \mathrm{mL}$ penicillin and $50 \mathrm{mg} / \mathrm{mL}$ streptomycin (Luneng antibacterial Lt. Co., China). Cells were sub-cultured 1:2 before reaching confluence using PBS and trypsin/EDTA (Gibco Life Technologies, USA) (Freshney, 1987).

Four pieces each of polarized $\left(d_{33}-100 \mathrm{pc} / \mathrm{N}\right)$ and unpolarized (negative control) ceramic samples were sterilized by autoclave and pretreated with culture media for 4 hours prior to cell implantation. Samples with 50\% (volume \%) AOM after $100 \mathrm{MaP}$ cold isostatic pressure were used in the in vitro study. Passage two isolated osteoblasts were seeded on the negative side of pretreated ceramics in 24-well

Table 1 Starting materials used for making porous LNKN ceramics

\begin{tabular}{lll}
\hline & \multicolumn{1}{c}{$\mathrm{LNKN}$} & \multicolumn{1}{c}{ Ammonium oxalate monohydrate (AOM) } \\
\hline Chemical composition & $\mathrm{Li}_{0.06} \mathrm{Na}_{0.5} \mathrm{~K}_{0.44} \mathrm{NbO}_{3}$ & $\left(\mathrm{NH}_{4}\right)_{2} \mathrm{C}_{2} \mathrm{O}_{4} \cdot \mathrm{H}_{2} \mathrm{O}$ \\
Particle size $(\mu \mathrm{m})$ & 0.97 & $250-300$ \\
Density $(\mathrm{g} / \mathrm{mL})$ & 4.255 & 1.50 \\
Decomposition point $\left({ }^{\circ} \mathrm{C}\right)$ & - & 75 \\
Source & Synthesized & Kelong Chemical Co., China \\
\hline
\end{tabular}

- 100 - International Journal of Oral Science, 1(2): 99-104, 2009 
cell culture plates at a density of $1 \times 10^{5}$ cells/ sample for MTT assay and at $5 \times 10^{5}$ cells/sample for SEM analysis. Cells were incubated in $1 \mathrm{~mL}$ of the same medium used for maintenance culture for the duration of the experiment. Four 24-well culture dishes without ceramics were used as positive controls for the MTT assay.

\section{Attachment and proliferation of osteoblasts}

SEM analyses were performed to study the morphology of osteoblasts grown for 72 hours on the surface of both test and negative control disks after cell seeding. The first step involved dehydration of the cells attached to the disk surface. The disks were placed in ethanol at $30 \%, 50 \%, 70 \%, 80 \%$, $90 \%, 95 \%$ and $100 \%$, immersed for 15 minutes at each step. The second step was to substitute ethanol with acetone (Kelong Chemical Co., China). After the critical point drying of these samples, SEM images were collected at $\times 1000$ and $\times 2000$ magnification on a Hitachi S-2460N SEM (Hitachi, Japan) to view the morphology of the cells attaching to the surfaces of the control and polarized disks.

Cell proliferation was assessed using MTT assay after growing cells in 24-well plates at a density of $1 \times 10^{5}$ cells/well for $1,3,5$ or 7 days. For the MTT assay, $10 \mu \mathrm{L}$ of MTT stock solution $(5 \mathrm{mg} / \mathrm{mL}$ of MTT in PBS) was added to each culture. After incubation for 4 hours, $100 \mu \mathrm{L}$ DMSO (Kelong Chemical Co., China) was added to each well and the plate was shaken evenly for 10 minutes. The solution was allowed to solubilize for another 30 minutes in the incubator. The absorbance of each well was determined using the Microplate Reader (Benchmark, Bio-Rad, USA) at $570 \mathrm{~nm}$.

\section{Statistical analysis}

Data are presented as means and standard errors of means. Statistical analysis was performed using one-way ANOVA for the MTT assay. $P$-values $<0.05$ were considered statistically significant.

\section{Results}

\section{Microstructure}

Stable 3-3 porous disks with average porosity of from about $47 \%-55 \%$ were generated using $\mathrm{AOM}$ as porogen under both compressive pressures. Figure 1 shows the XRD patterns of the ceramics under $20 \mathrm{MPa}$ conventional pressure and $100 \mathrm{MPa}$ cold isostatic pressures at 50\% (volume \%). Only one crystalline phase of perovskite structure was detected in each case.

Figure 2 shows SEM images of the surface topographies at $50 \%$ (volume \%) $\mathrm{AOM}$ at two different pressures. Pores $150-250 \mu \mathrm{m}$ in diameter were present, being somewhat smaller than the porogen particles. The pores were seen to be continuous. Large pores interconnected with each other through small pores and some small pores became incorporated into larger pores. In the walls of the pores $(\times 1000)$, micropores $1-3 \mu \mathrm{m}$ in diameter could be observed. In ceramics generated under low pressure, small cracks were evident in the matrix (Figures 2A and 2B) and the grains were only loosely in contact with each other which inhibited grain growth. However, in the ceramics generated under high pressure, the average grain size was about $10 \mu \mathrm{m}$ and the cubic-shaped grains were in close contact with each other (Figures $2 \mathrm{C}$ and 2D) leaving only some small micropores which served as channels for nutrition.

\section{Piezoelectric properties}

Both the $d_{33}$ and the mean $K_{\mathrm{p}}$ values decreased with increasing porosity (Table 2 ) owing to the volume fraction effect, which was consistent with previous reports (Gupta and Venkatesh, 2006; Wang et al., 2008). However, the ceramics with $50 \%$ porogens demonstrated piezoelectric properties relatively higher than the theoretical value $(-90 \mathrm{pC} / \mathrm{N})$ after CIP.

\section{Cell attachment}

Figure 3 shows SEM images of osteoblasts seeded on the surfaces of the porous LNKN after cell seeding for 72 hours on both polarized and unpolarized samples. The cells on the polarized sample covered $40 \%$ of the surface and appeared expanded with a well defined three-dimensional structure. However, cells on the surface without polarization showed less than $30 \%$ coverage and were flat, implying that osteoblasts had better cell behavior on the negative polarized surface than on the unpolarized surface.

\section{Cell proliferation}

Figure 4 shows the proliferation of osteoblasts on the surfaces of the porous LNKN and cell 


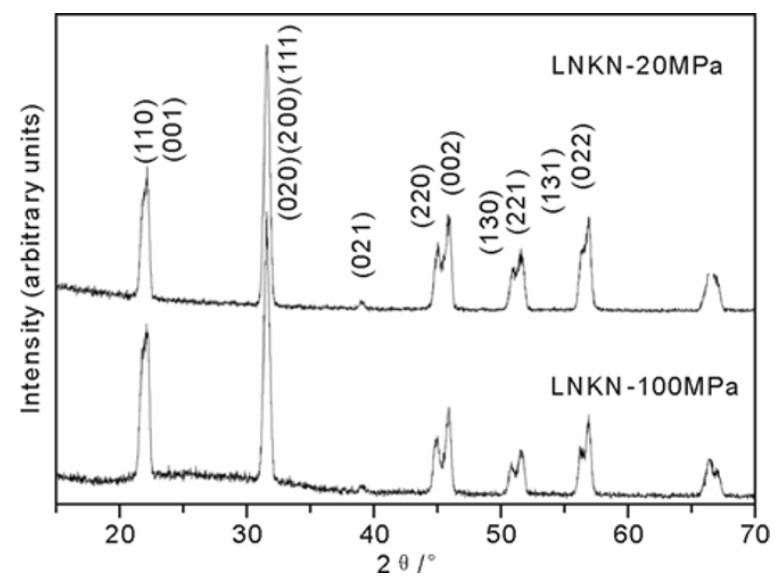

Figure 1 XRD patterns of the sintered LNKN porous ceramics
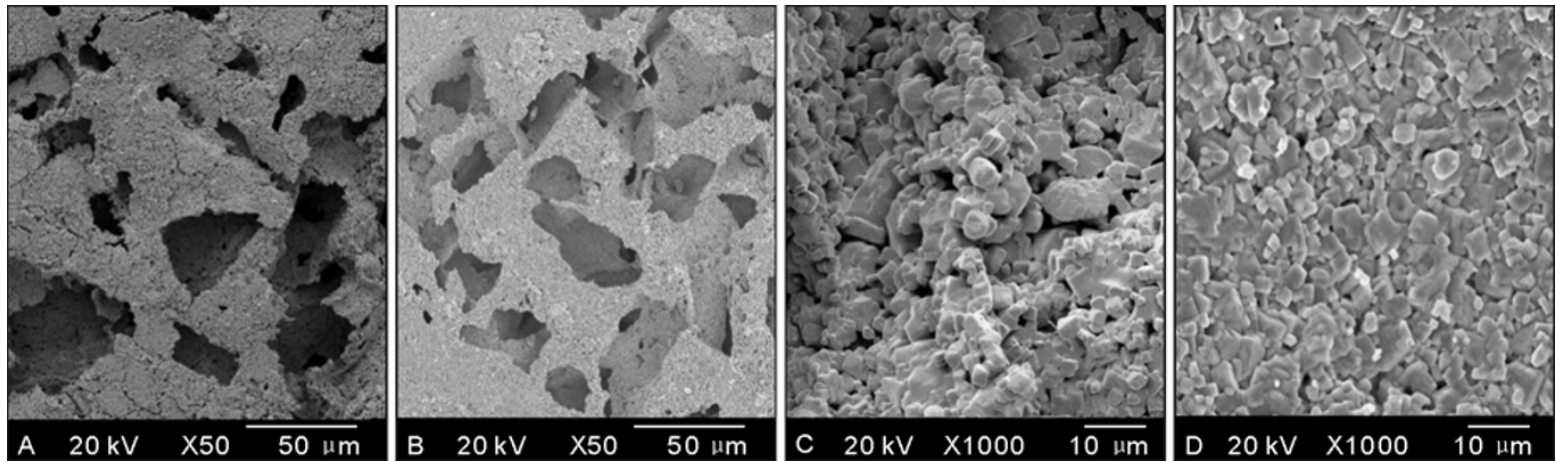

Figure 2 SEM images of the ceramics at 50\% AOM at different pressures

A, C: $20 \mathrm{MPa}$ cold isostatic pressure; B, D: $100 \mathrm{MPa}$ cold isostatic pressure.

Table 2 Piezoelectricity of the porous LNKN under two different compressive pressures $(\bar{\chi} \pm \mathrm{SD})$

\begin{tabular}{lcccc}
\hline & \multicolumn{2}{c}{$50 \%$ PFA } & \multicolumn{2}{c}{$60 \%$ PFA } \\
\cline { 2 - 5 } & $d_{33}$ & $K_{\mathrm{p}}$ & $d_{33}$ & $K_{\mathrm{p}}$ \\
\hline $20 \mathrm{Mpa}$ & $87 \pm 5$ & $0.23 \pm 0.02$ & $69 \pm 6$ & $0.18 \pm 0.03$ \\
$100 \mathrm{Mpa}$ & $117 \pm 6$ & $0.24 \pm 0.03$ & $76 \pm 8$ & $0.23 \pm 0.02$ \\
\hline
\end{tabular}

culture plates on days 1, 3, 5 and 7 after cell seeding. The living cells showed more proliferation on the polarized than on the unpolarized porous surfaces. The proliferation of the cells on the polarized specimen surfaces was greater on days 3, 5 and 7 $(P<0.05)$ which is consistent with the SEM results. This indicated that both polarized and unpolarized lead-free piezoelectric ceramic samples did not show cytotoxicity during the testing period.

\section{Discussion}

Piezoelectric materials have made contributions to many different fields (Chen et al., 2004). Their first application to the biomaterials area was with the discovery of lead-free piezoelectric ceramic barium titanate $\left(\mathrm{BaTiO}_{3}\right)$ (Park et al., 1980). Lithium sodium potassium niobate has broadened the scope of lead-free piezoelectric ceramics owing to its better and more stable piezoelectricity than barium titanate (Saito et al., 2004). In this study, the manufacturing method for porous LNKN ceramics was modified by CIP, a method for consolidation under a uniform pressure in a flexible envelope. The process was applied to pre-shaped LNKN-AOM samples resulting in a high degree of uniformity in density after sintering.

The SEM images of the ceramics at two different pressures were compared and no defects or flaws were evident in the walls of the pores generated by CIP (Figures 2A and 2B). This is because high pressure makes the matrix much firmer and stiffer compared to conventional pressure. CIP-generated ceramics consisted of fine and coarse grains and had a smaller grain size than ordinary ceramics (Figures 2C and 2D), indicating that grain growth was promoted by CIP. Porous ceramics can be regarded as pore-solid composites with pores being the second phase. Those with independent pores can be classified as $0-3$ and those with continuous 

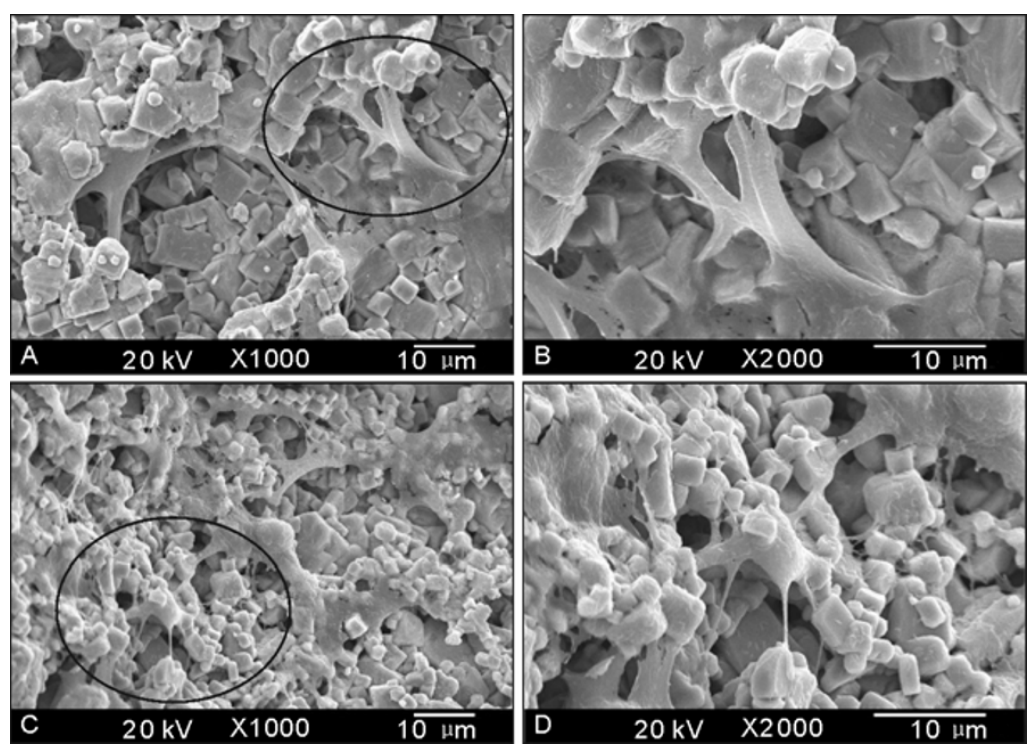

Figure 3 SEM images of osteoblasts seeded on the surfaces of the porous LNKN after cell seeding for 72 hours on both polarized and unpolarized samples

A, C: 20 MPa cold isostatic pressure; B, D: 100 MPa cold isostatic pressure.



Figure 4 MTT assay of proliferation of osteoblasts cultured on different substrates

Error bar represent $\bar{\chi} \pm \mathrm{SD}, n=4, * P<0.05$.

pores as 3-3 (Della et al., 2006). Interconnected pores could be observed in the SEM images of the ceramics after CIP, indicating that a 3-3 porous pore-solid structure was obtained with pore sizes favorable for cell growth and nutrition exchange.

The piezoelectric properties of 3-3 composites can be predicted by theoretical models (Banno, 1987) and as previously reported, those of porous LNKN closely matched theoretical models (Wang et al., 2008). However, in this study, the samples with $50 \%$ porogens demonstrated a relatively higher $d_{33}$ value than the theoretical value $(-90 \mathrm{pC} / \mathrm{N})$ after CIP. The reason may be that the crystals grew better after CIP and the increased stiffness of the matrix enabled the electric domain to reverse more easily, further increasing the piezoelectric constant. Therefore, the CIP procedure compensates for the reduction in piezoelectricity caused by the modified polarization condition necessary for biocompatibility (Wang et al., 2008).

In vitro results showed that the lead-free piezoelectric ceramic samples had no cytotoxicity during the testing period. As shown previously, the $\mathrm{pH}$ value of ceramics in culture media remains stable for up to 5 months. Therefore, it could be predicted that no changes would occur in the extracellular environment if the ceramics were implanted for a longer period (Wang et al., 2008). Osteogenesis is thought to be promoted by piezoelectric material. Fundamentally, new bone formation is mediated by pressure-generated electrical current and surface electronic charges (Zhang et al., 2007). Furthermore, new bone formation depends on the piezoelectric constants (Braden et $a l .$, 1966). $d_{33}$ is the polarization generated per unit of mechanical stress and $K_{\mathrm{p}}$ is the factor for the electric field in the direction parallel to which a ceramic is polarized (Du et al., 2007). In living beings, polar materials like collagen, ions and active cells grow in a definite direction. Cells were more active on negative polarized surfaces than on unpolarized ones because of the electronic cellular reaction between osteoblasts and the electric domain both on the surface and inside the pores. The range in which cells will exhibit their best behavior for 
osteogenesis is still unknown. However, based on our findings, the osetoblasts worked well on the best polarized surface. Further studies are needed to determine the most optimum surface. Long-term and animal studies are also needed to verify the osteoinductive properties and in vivo status of the new porous lead-free piezoelectric ceramics.

\section{Conclusion}

Lead-free piezoelectric ceramics with 3-3 porous structure with the potential for use as bone substitutes can be generated using AOM. Cold isostatic pressure of $100 \mathrm{MPa}$ may enhance their piezoelectric properties by improving their inert structure. The positive effects shown in our in vitro study on the attachment and differentiation of SD osteoblasts on porous materials demonstrated good cytocompatibility, indicating that piezoelectric composites show promise for use as bone substitutes.

\section{Acknowledgements}

This work was financially supported by the National Natural Science Foundation of China (59972020).

\section{References}

Banno H (1987). Effects of shape and volume fraction of closed pores on dielectric, elastic, and electromechanical properties of dielectric and piezoelectric ceramics: a theoretical approach. Ceram Bull, 66(9): 1332-1337.

Braden M, Bairstow A, Beider I, Ritter B (1966). Electrical and piezoelectrical properties of dental hard tissues. Nature, 212: 1565-1566.

Chen Q, Chen L, Li Q, Yue X, Xiao DQ, Zhu JG (2007). Piezoelectric properties of $\mathrm{K}_{4} \mathrm{CuNb}_{8} \mathrm{O}_{23}$ modified $\left(\mathrm{Na}_{0.5} \mathrm{~K}_{0.5}\right) \mathrm{NbO}_{3}$ lead-free piezoceramics. $J$ Applied Phys, 102(10): 104-109.

Chen YC, Wu S (2004). Piezoelectric composites with 3-3 connectivity by injecting polymer for hydrostatic sensors. Ceramics Inter, 30(1): 69-74.

Della CN, Shu D (2006). The performance of 1-3 piezo- electric composites with a porous non-piezoelectric matrix. Acta Mater, 56(4): 754-761.

Du HL, Tang F, Luo F, Zhou W, Qu S, Pei Z (2007). Effect of poling condition on piezoelectric properties of $\left(\mathrm{K}_{0.5} \mathrm{Na}_{0.5}\right) \mathrm{NbO}_{3}-\mathrm{LiNbO}_{3}$ lead-free piezoelectric ceramics. Materials Science and Engineering B, 137 (1-3): 175-179.

Feng J, Yuan H, Zhang X (1997). Promotion of osteogenesis by a piezoelectric biological ceramic. Biomaterials, 18(23): 1531-1534.

Freshney RI (1987). Culture of animal cells: a manual of basic technique. New York: Alan R. Liss, Inc., pp.275-277.

Gupta RK, Venkatesh TA (2006). Electromechanical response of porous piezoelectric materials. Acta Materialia, 54(15): 4063-4078.

Miara B, Rohan E, Zidi M, Labat B (2005). Piezomaterials for bone regeneration design-homogenization approach. J Mecha Physic Solids, 53(11): 2529-2556.

Park JB, Recum AF, Kenner GH, Kelly BJ (1980). Piezoelectric ceramic implants: a feasibility study. $J$ Biomed Mater Res, 14(3): 269-277.

Park JB, Kelly BJ, Kenner GH, Recum AF (1981). Piezoelectric ceramic implants: in vivo results. $J$ Biomed Mater Res, 15(1): 103-110.

Saito Y, Takao H, Tani T, Nonoyama T, Takatori K, Homma T, et al. (2004). Lead-free piezoceramics. Nature, 432(7013): 84-87.

Wang P (2006). Study on lithium sodium potassium niobate novel biological piezoelectric ceramic. Chengdu, China: Sichuan University.

Wang PY, Zhang XZ, Yang P, Tian BM, Liu ZL, Chen ZQ (2008). Biocompatibility if a novel biological piezoelectric ceramic to the rat periosteum derived osteobalst. West China J Stoma, 26(2): 133-137.

Wang Q, Chen Q, Zhu J, Huang C, Darvell BW, Chen Z (2008). Effects of pore shape and porosity on the properties of porous LNKN ceramics as bone substitute. Mater Chem and Physic, 109(2-3): 488-491.

Wang Q, Chen X, Zhu J, Darvell BW, Chen Z (2008). Porous $\mathrm{Li}-\mathrm{Na}-\mathrm{K}$ niobate bone-substitute ceramics: microstructure and piezoelectric properties. Mater Letter, 62(20): 3506-3508.

Zhang H, Li J, Zhang B (2007). Microstructure and electrical properties of porous PZT ceramics derived from different pore-forming agents. Acta Mater, 55(1): 171.

\footnotetext{
*Corresponding author: Zhi-qing Chen

Address: State Key Laboratory of Oral Diseases, West China College of Stomatalogy, Sichuan University, No.14, $3^{\text {rd }}$ Section, Renmin South Road, Chengdu 610041, China

Tel: $862885502407 \quad$ Fax: $862885502401 \quad$ Email: ChenZhiQing40@126.com
} 